\title{
Heavy rotation - evolution of quadrupole collectivity centred at the neutron-rich doubly mid-shell nucleus ${ }^{170}$ Dy
}

Cite as: AIP Conference Proceedings 1681, 030010 (2015); https://doi.org/10.1063/1.4932254

Published Online: 15 October 2015

P.-A. Söderström, P. H. Regan, P. M. Walker, H. Watanabe, P. Doornenbal, Z. Korkulu, P. Lee, H. L. Liu, J. J. Liu, G. Lorusso, S. Nishimura, T. Sumikama, V. H. Phong, J. Wu, F. R. Xu, A. Yagi, G. X. Zhang, T. Alharbi, H. Baba, F. Browne, A. M. Bruce, R. Carroll, K. Y. Chae, Zs. Dombradi, A. Estrade, N. Fukuda, C. Griffin, E. Ideguchi, N. Inabe, T. Isobe, H. Kanaoka, I. Kojouharov, F. G. Kondev, T. Kubo, S. Kubono, N. Kurz, I. Kuti, S. Lalkovski, G. J. Lane, C. S. Lee, E. J. Lee, G. Lotay, C.-B. Moon, I. Nishizuka, C. R. Nita, A. Odahara, Z. Patel, Zs. Podolyák, O. J. Roberts, H. Sakurai, H. Schaffner, C. M. Shand, H. Suzuki, H. Takeda, S. Terashima, Zs. Vajta, J. J. Valiente-Dòbon, Z. Y. Xu, and S. Yoshida

\section{ARTICLES YOU MAY BE INTERESTED IN}

Status and results from the decay spectroscopy project EURICA (Euroball-RIKEN cluster array) AIP Conference Proceedings 1753, 070001 (2016); https://doi.org/10.1063/1.4955364

Decay spectroscopy of neutron-rich rare-earth isotopes and collectivity around double midshell

AIP Conference Proceedings 1681, 030009 (2015); https://doi.org/10.1063/1.4932253

Half-life measurements of isomeric states populated in projectile fragmentation AIP Conference Proceedings 1491, 317 (2012); https://doi.org/10.1063/1.4764266

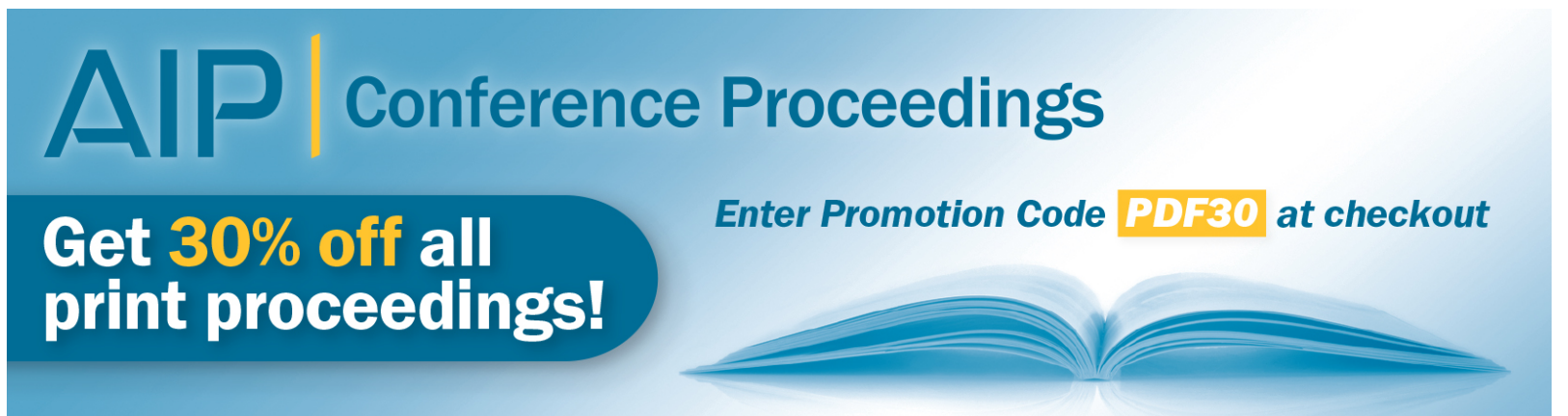




\section{Heavy Rotation - Evolution of quadrupole collectivity centred at the neutron-rich doubly mid-shell nucleus ${ }^{170} \mathrm{Dy}$}

P.-A. Söderström ${ }^{1, \text { a) }}$, P. H. Regan ${ }^{2,3}$, P. M. Walker ${ }^{2}$, H. Watanabe ${ }^{4,5}$, P. Doornenbal ${ }^{1}$, Z. Korkulu ${ }^{6}$, P. Lee ${ }^{7}$, H.L. Liu ${ }^{8}$, J.J. Liu ${ }^{9}$, G. Lorusso ${ }^{1,3}$, S. Nishimura ${ }^{1}$, T. Sumikama ${ }^{10}$, V. H. Phong ${ }^{1,11}$, J. Wu ${ }^{1,12}$, F.R. Xu ${ }^{12}$, A. Yagi ${ }^{13}$, G.X. Zhang ${ }^{5}$, T. Alharbi ${ }^{14}$, H. Baba ${ }^{1}$, F. Browne ${ }^{15}$, A.M. Bruce ${ }^{14}$, R. Carroll ${ }^{2}$, K.Y. Chae ${ }^{16}$, Zs. Dombradi ${ }^{6}$, A. Estrade ${ }^{17}$, N. Fukuda ${ }^{1}$, C. Griffin ${ }^{17}$, E. Ideguchi ${ }^{13,18}$, N. Inabe ${ }^{1}$, T. Isobe ${ }^{1}$, H. Kanaoka ${ }^{13}$, I. Kojouharov ${ }^{19}$, F.G. Kondev ${ }^{20}$, T. Kubo ${ }^{1}$, S. Kubono ${ }^{1}$, N. Kurz ${ }^{19}$, I. Kuti ${ }^{6}$, S. Lalkovski ${ }^{2}$, G. J. Lane ${ }^{21}$, C.S. Lee ${ }^{7}$, E.J. Lee ${ }^{16}$, G. Lotay ${ }^{2}$, C.-B. Moon ${ }^{22}$, I. Nishizuka ${ }^{10}$, C.R. Nita ${ }^{15,23}$, A. Odahara ${ }^{13}$, Z. Patel ${ }^{2}$, Zs. Podolyák ${ }^{2}$, O.J. Roberts ${ }^{24}$, H. Sakurai ${ }^{1,25}$, H. Schaffner ${ }^{19}$, C.M. Shand ${ }^{2}$, H. Suzuki ${ }^{1}$, H. Takeda ${ }^{1}$, S. Terashima ${ }^{5}, Z^{2}$. Vajta ${ }^{6}$, J.J. Valiente-Dòbon ${ }^{26}$, Z.Y. Xu ${ }^{9}$ and $\mathrm{S}$. Yoshida ${ }^{13}$

\footnotetext{
${ }^{1}$ RIKEN Nishina Center, 2-1 Hirosawa, Wako-shi, Saitama 351-0198, Japan

${ }^{2}$ Department of Physics, University of Surrey, Guildford, GU2 7XH, UK

${ }^{3}$ National Physical Laboratory, Teddington, Middlesex, TW11 OLW, UK ${ }^{4}$ IRCNPC, Beihang University, Beijing 100191, China

${ }^{5}$ School of Physics and Nuclear Energy Engineering, Beihang University, Beijing 100191, China ${ }^{6}$ Institute for Nuclear Research, Hungarian Academy of Sciences, P. O. Box 51, Debrecen, H-4001, Hungary

${ }^{7}$ Department of Physics, Chung-Ang University, Seoul 156-756, Korea

${ }^{8}$ Department of Applied Physics, School of Science, Xi'an Jiaotong University, Xi'an 710049, China

${ }^{9}$ Department of Physics, the University of Hong Kong, Pokfulam Road, Hong Kong

${ }^{10}$ Department of Physics, Tohoku University, Aoba, Sendai, Miyagi 980-8578, Japan

${ }^{11}$ VNU Hanoi University of Science, 334 Nguyen Trai, Thanh Xuan, Hanoi, Vietnam

${ }^{12}$ School of Physics, Peking University, Beijing 100871, China

${ }^{13}$ Department of Physics, Osaka University, Machikaneyama-machi 1-1, Osaka 560-0043 Toyonaka, Japan

${ }^{14}$ Department of Physics, College of Science in Zulf, Almajmaah University, P.O. Box 1712, 11932, Saudi Arabia

${ }^{15}$ School of Computing, Engineering and Mathematics, University of Brighton, Brighton BN2 4JG, UK

${ }^{16}$ Department of Physics, Sungkyunkwan University, Suwon 440-746, Korea

${ }^{17}$ School of Physics and Astronomy, University of Edinburgh, Edinburgh EH9 3JZ, UK

${ }^{18}$ Research Center for Nuclear Physics (RCNP), Osaka University, Ibaraki, Osaka 567-0047, Japan

${ }^{19}$ GSI Helmholtzzentrum für Schwerionenforschung GmbH, 64291 Darmstadt, Germany

${ }^{20}$ Nuclear Engineering Division, Argonne National Laboratory, Argonne, Illinois 60439, USA

${ }^{21}$ Department of Nuclear Physics, R.S.P.E., Australian National University, Canberra, A.C.T. 0200, Australia

${ }^{22}$ Hoseo University, Asan, Chungnam 336-795, Korea

${ }^{23}$ Horia Hulubei National Institute of Physics and Nuclear Engineering, RO-077125 Bucharest, Romania

${ }^{24}$ School of Physics, University College Dublin, Belfield, Dublin 4, Ireland

${ }^{25}$ Department of Physics, University of Tokyo, Hongo, Bunkyo-ku, Tokyo 113-0033, Japan

${ }^{26}$ Istituto Nazionale di Fisica Nucleare, Laboratori Nazionali di Legnaro, 35020 Legnaro, Italy

${ }^{a)}$ Corresponding author: pasoder@ ribf.riken.jp
} 
Abstract. In this contribution the low-excitation structural properties of the doubly mid-shell nucleus ${ }^{170}$ Dy are discussed, with a special empasis on the evolution of the ground state rotational band within the dysprosium isotopic chain. Recent results from an experiment with the EURICA setup at RIKEN are shown in the context of previous measurements at the PRISMA+CLARA as well as the PRISMA+AGATA setups at Laboratori Nazionali di Legnaro. A brief outlook on future planned measurements is also given.

\section{INTRODUCTION}

One of the most successful descriptions of the structure of nuclei is the nuclear shell model. However, far from the closed shells it is the interplay between the macroscopic shape degrees of freedom and the microscopic nature of the underlying single-particle orbitals in the deformed basis that offers an explanation for the nuclear behaviour. Lying precisely in the middle of the the closed proton $Z=50,82$ and neutron $N=82,126$ shells, the $Z=66$ and $N=104$ nucleus ${ }^{170}$ Dy has for a long time been somewhat of a "holy grail" for the collective models of nuclear physics. However, the shape evolution can also be influenced by the presence of sub-shell closures and residual interactions dependent on the neutron excess [1]. Thus, understanding of the neutron-rich Dy region would be useful for testing competing nuclear mean-field model calculations of nuclear shapes $[2,3,4]$.

Recently several experiments have been performed aiming for ${ }^{170} \mathrm{Dy}$. In these proceedings, we will focus on the evolution of the ground state band in the context the discussion provided in the previous experimental work on this nucleus [5].

\section{BACKGROUND}

The first experiment that successfully observed excited states in ${ }^{170}$ Dy was carried out at the PRISMA and CLARA set-up [6] in May 2007 at the LNL accelerator complex using multi-nucleon transfer reactions between ${ }^{82} \mathrm{Se}$ and ${ }^{170} \mathrm{Er}$. By using two-body kinematics it was possible to reconstruct the $A, Z$, and the velocity vector of the target-like fragments before neutron evaporation. Together with neutron-evaporation suppression techniques based on time-offlight very clean spectra of the target-like fragments were obtained. In this experiment, the yrast band of ${ }^{168}$ Dy was observed up to $10^{+}$and a tentative identification of the $4^{+} \rightarrow 2^{+}$transition in ${ }^{170} \mathrm{Dy}$ at $163 \mathrm{keV}$ was obtained for the fist time [5].

This study was followed up in October 2011 [7] with an experiment using the AGATA [8] setup together with the PRISMA spectrometer. This time, a ${ }^{136} \mathrm{Xe}$ beam was used on a ${ }^{170} \mathrm{Er}$ target to populate ${ }^{168} \mathrm{Dy},{ }^{170} \mathrm{Dy}$, and ${ }^{172} \mathrm{Dy}$, respectively. According to GRAZING calculations, including neutron evaporation, the use of a ${ }^{136} \mathrm{Xe}$ beam instead of an ${ }^{82} \mathrm{Se}$ beam would give a large increase of the yield of the neutron-rich reaction products [9] as well as of the angular momentum transferred to the fragments. In that experiment, the DANTE detector array [10] was included for additional channel selection using delayed $\gamma$ rays in AGATA. Besides the possibility to tag on the predicted $6^{+}$isomer in ${ }^{170} \mathrm{Dy}$ itself, it would be possible to identify ${ }^{168,170} \mathrm{Dy}$ from the $10^{+}$isomers in the binary partners ${ }^{136} \mathrm{Ba}$ and ${ }^{134} \mathrm{Ba}$. The data from this experiment is currently under analysis by the Uppsala group [11].

As a next step, with the high-intensity fission beams available at the Radioactive Isotope Beam Factory (RIBF) at RIKEN, it is natural to pursue this topic at this facility. An isotope search experiment in October 2011 proved the feasibility of a dedicated decay experiment in this region. Together, the experimental cross-sections as well as the existence of an isomeric state provided valuable information for a detailed study of the structure of ${ }^{170} \mathrm{Dy}$ [12].

\section{RIKEN EXPERIMENT}

From December 2011, 84 high-purity germanium crystals were delivered from GSI to the RIBF. These crystals were assembled into the Euroball-RIKEN Cluster Array (EURICA) [13,14] and installed at the end of the BigRIPS and ZeroDegree beam-line $[15,16]$ into a decay spectroscopy setup. In April 2012, the setup was successfully commissioned [14] and since then a number of large data sets have been collected focused on different regions of the Segré chart. The first experiment with a uranium beam was performed in December 2012 in the ${ }^{78} \mathrm{Ni}$ region [17] and since then $\beta$-decay data have been collected covering most of the neutron-rich part of the nuclear chart up to the rare-earth region $[18,19,20]$. In parallel to the decay data, isomer decay information has been collected reaching all the way up to ${ }^{166} \mathrm{Gd}[21]$. 

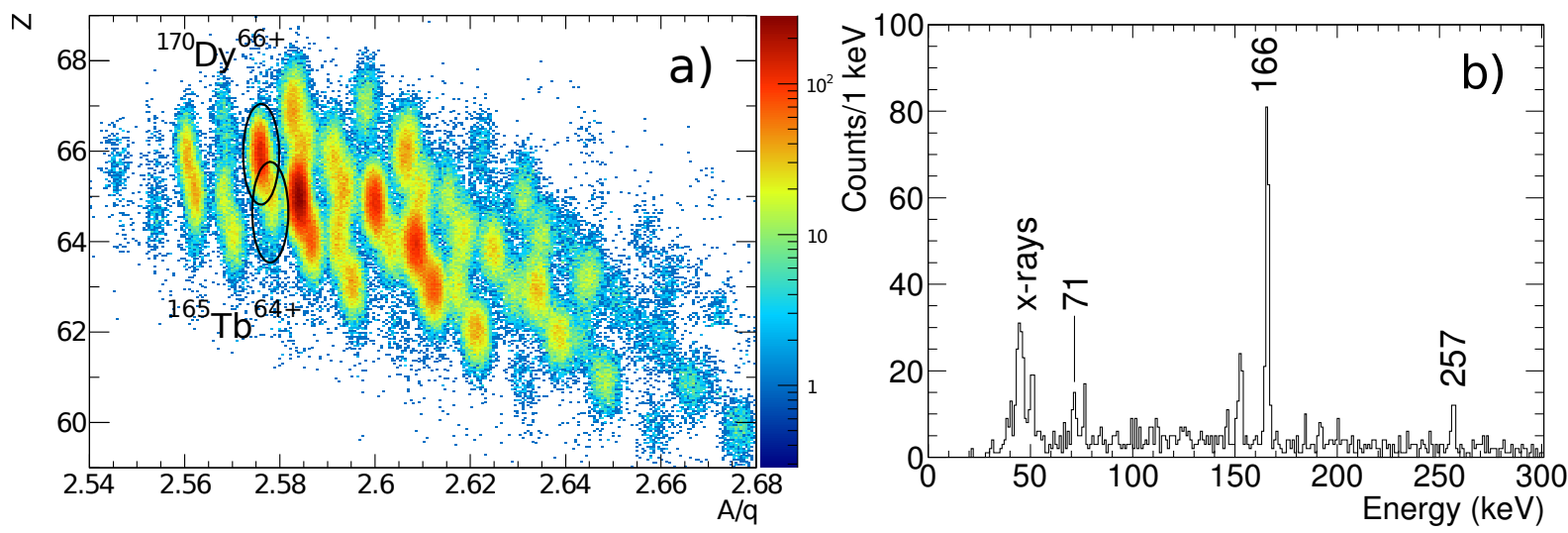

FIGURE 1. Particle identification of the nuclei produced in the RIKEN experiment (left) and the low-energy $\gamma$-ray spectrum associated with the isomer decay of ${ }^{170} \mathrm{Dy}$ (right).

In these experiments, the radioactive isotopes are typically produced by in-flight fission of a $345 \mathrm{MeV} / \mathrm{u}{ }^{238} \mathrm{U}$ beam on a beryllium target with beam intensities around $10 \mathrm{pnA}$. The secondary beams are then separated and identified using the BigRIPS and ZeroDegree spectrometers on an event-by-event basis by their mass-to-charge ratio $(A / q)$ and atomic number $(Z)$, before being implanted in the WAS3ABi active stopper [13, 22, 23]. This stopper consists of up to eight $40 \times 60 \mathrm{~mm}^{2}$ double-sided silicon-strip detectors with strip widths of $1 \mathrm{~mm}$ in horizontal and vertical direction.

In November 2014, the experiment aimed at identifying the low-lying structures in ${ }^{170}$ Dy was performed. In this experiment as well as during the experiment aiming at ${ }^{110} \mathrm{Zr}$ in spring 2013, an array of $18 \mathrm{LaBr}_{3}$ :Ce detectors [24, 25] was mounted in three empty positions of the EURICA support structure for fast timing measurements [26, 27]. The experiment was carried out with two settings with 13.5 hours focusing on ${ }^{170} \mathrm{Dy}(\sim 10000$ implantations $)$ and 45 hours during which $\sim 2500{ }^{170} \mathrm{~Tb}$ nuclei, the $\beta$-decay mother of ${ }^{170} \mathrm{Dy}$, were implanted. The particle identification plot from this experiment is shown in Fig. 1a. For isomer decays, EURICA was triggered by a thin plastic scintillator at the end of the beam line and read out for a time window of $100 \mu \mathrm{s}$ after the passing of an ion. For $\beta$-decay events the read-out was triggered by an electron signal in WAS3ABi. The correlation between ion implantation and $\beta$-delayed $\gamma$-rays was constructed based on an electron signal within $2 \mathrm{~mm}$ of the implanted ion. Due to the high contamination from lighter fragments, a plastic veto detector was placed behind WAS3ABi for ions passing through the silicon detector.

The low energy part of the isomer decay spectrum is shown in Fig. 1b. The $\gamma$-ray spectrum obtained during a time window of $0.3-6 \mu \mathrm{s}$ after the implantation of ${ }^{170} \mathrm{Dy}$. Peaks belonging to ${ }^{170} \mathrm{Dy}$ have been labeled according to their $\gamma$-ray energy, while unlabeled peaks have been identified to originate from $\mathrm{H}$-like charge states of ${ }^{165} \mathrm{~Tb}$ nuclei, which were not fully separated in $Z$. The ground state rotational band of ${ }^{170} \mathrm{Dy}$ can clearly be seen up to the $6^{+}$state, as shown in Fig. 2, with an energy ratio between the first $4^{+}$and $2^{+}$states, $R(4 / 2)=3.321(7)$. This ratio is consistent with ${ }^{168}$ Dy that has the experimental $R(4 / 2)=3.3128(30)$. Despite the increase being small, it is the highest value found in this region and it implies that ${ }^{170}$ Dy is very close to the idealised rotor. This means that the $E\left(2^{+}\right)$should indeed reflect the deformation suggesting that, contrary to Refs. $[3,4,28]$, the deformation maximum in the isotopic chain is located at $N=104$. This picture should, however, be confirmed by more direct measurements mentioned in the outlook.

\section{GROUND STATE BAND SYSTEMATICS}

Self-consistent Hartree-Fock calculations with a variety of Skyrme parametrizations all suggest that the deformation maximum is expected at either ${ }^{166}$ Dy or ${ }^{168}$ Dy [3]. This is also consistent with one of the standard references for nuclear masses and deformations, the calculations made by Möller and Nix using the finite range liquid drop model [28], where ${ }^{168}$ Dy has the largest value. However, the decrease in $E\left(2^{+}\right), E\left(4^{+}\right)$, as well as the increase of $R(4 / 2)$ suggest that the collectivity and deformation continue to increase at least until $N=104$. This is more consistent with the naïve neutron mid-shell picture rather than the more comprehensive nuclear models. 


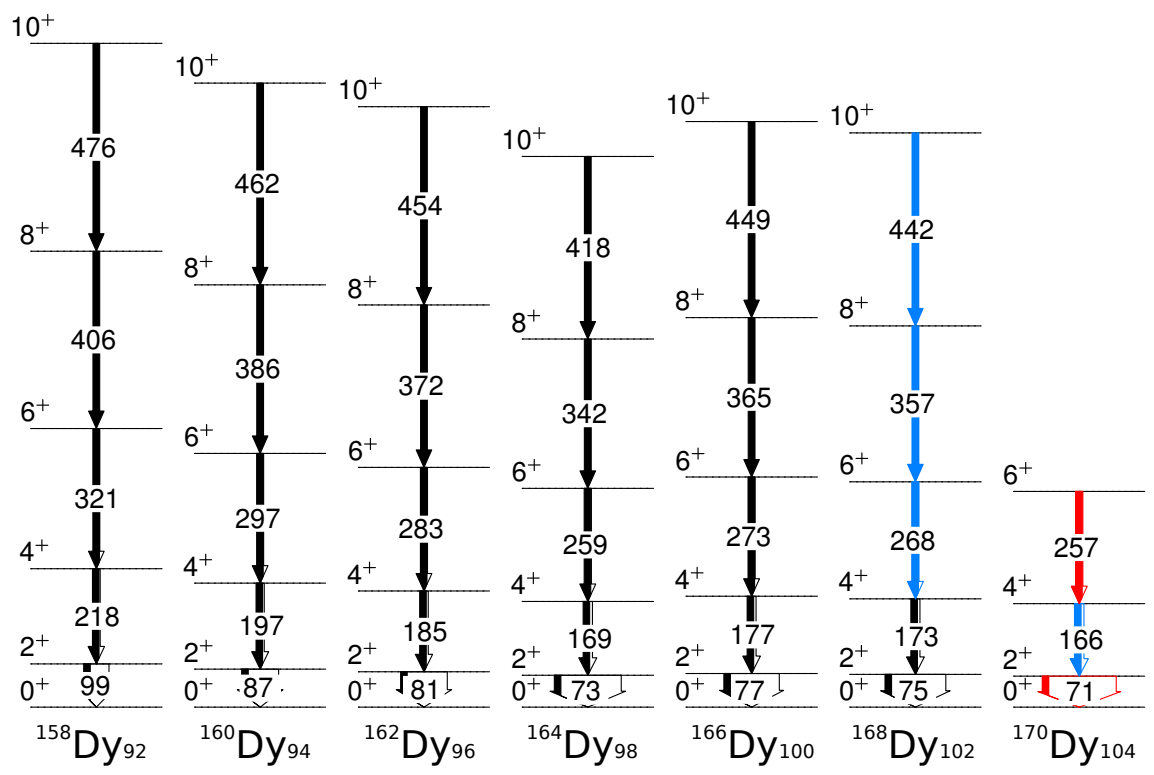

FIGURE 2. Systematics of the yrast bands in the dysprosium chain up to the $10^{+}$state. Data from Ref. [5] are marked in blue and RIKEN experimental results is marked in red.

Another interesting feature is the irregularity at $N=98$. This irregularity is not reproduced by Total Routhian Surface (TRS) calculations [29, 30, 31] shown in Fig. 3 and is yet to be explained, although recent experimental results suggest a connection to a possible deformed shell closure at $N=100$ [21]. It is also worth noting that the TRS calculations underestimate the moment of inertia of ${ }^{170} \mathrm{Dy}$ and suggest a maximum at lower values of $N$, consistent with other theoretical work. The interpretation that the irregularity is an effect at $N=98,100$ and not in neighboring isotopes is strengthened by the identification of the $6^{+}, 4^{+}$, and $2^{+}$transitions in ${ }^{170} \mathrm{Dy}$.

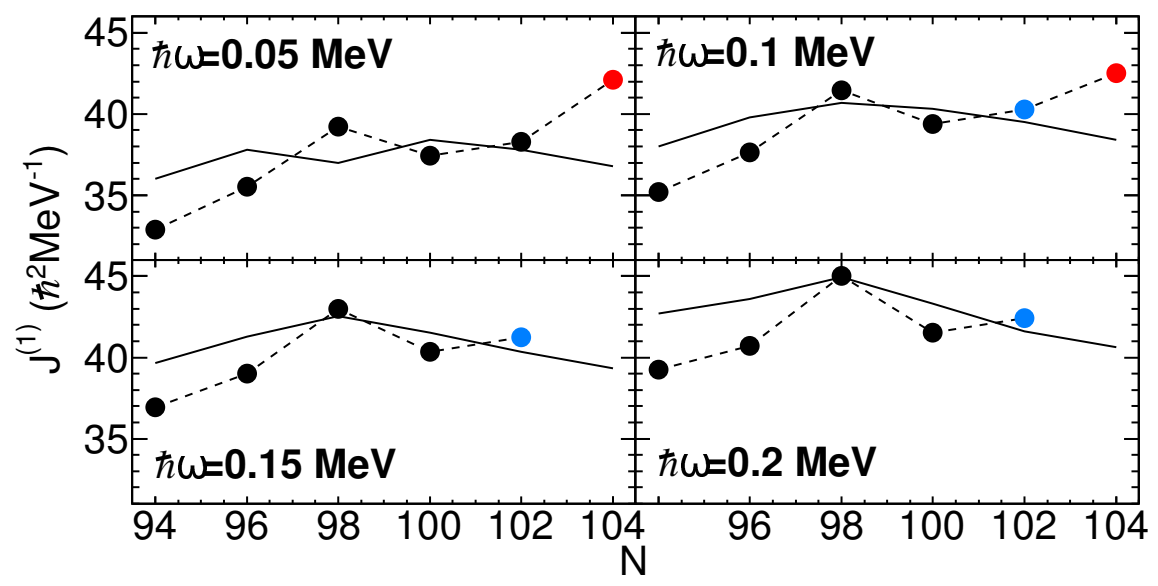

FIGURE 3. Experimental (circles) and calculated [5] (solid line) moments of inertia at a given rotational frequency of $\hbar \omega=0.05$, $0.10,0.15$ and $0.20 \mathrm{MeV}$ for dysprosium isotopes with $N=94-104$. The experimental values are obtained using linear interpolation between measured rotational frequencies. Data from Ref. [5] are marked in blue and RIKEN experimental results is marked in red. 


\section{OUTLOOK}

In these proceedings the first results of the ground state structure in ${ }^{170} \mathrm{Dy}$ were presented from measurements with CLARA and EURICA. Completing the analysis of the experiments mentioned here will provide more detailed information about the $\gamma$ deformation, single particle structure, octupole deformation, and the evolution of the yrast band at higher spins. This may help solving some of the questions currently unanswered about the structure evolution around mid-shell. In addition, future experiments may shed more light on the underlying physics. While the $E\left(2^{+}\right)$and $R(4 / 2)$ observables were discussed here, for a complete picture the $B(\mathrm{E} 2)$ values have a strong dependency on the evolution of collectivity. Such information can be obtained with the ROSPHERE array [32, 33] that has previously been used successfully in this region [34], or with a fast timing campaign using AGATA at GANIL [35].

\section{REFERENCES}

[1] R. B. Cakirli, et al., Phys. Rev. Lett. 82, 061302 (2010).

[2] P. H. Regan, et al. Phys. Rev. C 65, 037302 (2002).

[3] A. K. Rath, et al., Phys. Rev. C 68, 044315 (2003).

[4] C. E. Vargas, et al., Eur. Phys. J. A49, 4 (2013).

[5] P.-A. Söderström, et al., Phys. Rev. C 81, 034310 (2010).

[6] A. Gadea, et al., Eur. Phys. J. A20, 193 (2003).

[7] P.-A. Söderström, J. Nyberg, A. Ataç, P. H. Regan, et al., (2011), INFN-LNL proposal.

[8] S. Akkoyun, et al., Nucl. Instrum. Meth. A668, 26 (2012).

[9] P.-A. Söderström, Phys. Scr. T150, 014038 (2012).

[10] J. J. Valiente-Dobón, et al., in LNL Annual Report 2005, edited by D. R. Napoli, et al., Legnaro, Italy, 2006, p. 175.

[11] A. Gengelbach, Private communication.

[12] D. Kameda, et al., RIKEN Accel. Prog. Rep. 47, viii (2014).

[13] S. Nishimura, Prog. Theor. Exp. Phys. 2012, 03 C006 (2012).

[14] P.-A. Söderström, et al., Nucl. Instrum. Meth. B317, 649 (2013).

[15] T. Kubo, et al., Prog. Theor. Exp. Phys. 2012, 03 C003 (2012).

[16] N. Fukuda, et al., Nucl. Instrum. Meth. B317, 323 (2013).

[17] Z. Y. Xu, et al., Phys. Rev. Lett. 113, 032505 (2014).

[18] J. Wu, et al., AIP Conf. Proc. 1594, 388 (2014).

[19] J. Wu, et al., in Proceedings of XIII Nuclei in the Cosmos PoS (NIC XIII), p. 016 (2014).

[20] J. Wu, et al., JPS Conf. Proc. 6, 030064 (2015).

[21] Z. Patel, et al., Phys. Rev. Lett. 113, 262502 (2014).

[22] S. Nishimura, et al., RIKEN Accel. Prog. Rep. 46, 182 (2013).

[23] J. J. Liu, et al., RIKEN Accel. Prog. Rep. 48 (2015), In print.

[24] P. H. Regan, et al., EPJ Web Conf. 63, 01008 (2013).

[25] Z. Patel, et al., RIKEN Accel. Prog. Rep. 47, 13 (2014).

[26] F. Browne, et al., Acta Phys. Pol. 46721 (2015)

[27] F. Browne, et al., JPS Conf. Proc. 6030012 (2015)

[28] P. Moller, et al., At. Data Nucl. Data Tab. 59, 185 (1995).

[29] F. R. Xu, et al., Phys. Lett. B435, 257 (1999).

[30] F. R. Xu, et al., Phys. Rev. C 59, 731 (1999).

[31] F. R. Xu, et al., Phys. Rev. C 62, 014301 (2000).

[32] N. Mărginean, et al., Eur. Phys. J. A46, 329 (2010).

[33] P.-A. Söderström, et al., (2013), IFIN-HH proposal.

[34] P. J. R. Mason, et al., Phys. Rev. C 88, 044301 (2013).

[35] P. H. Regan, J. Nyberg, J. Simpson, et al., (2014), GANIL proposal, E705. 